\title{
Epilepsy surgery failure in children: a quantitative and qualitative analysis
}

Dario J. Englot, M.D., Ph.D. ${ }^{1,2}$, Seunggu J. Han, M.D. ${ }^{1,2}$, John D. Rolston, M.D., Ph.D. ${ }^{1,2}$, Michael E. Ivan, M.D. ${ }^{1,2}$, Rachel A. Kuperman, M.D. ${ }^{5}$, Edward F. Chang, M.D. ${ }^{1,2}$, Nalin Gupta, M.D., Ph.D. ${ }^{1,2,4}$, Joseph E. Sullivan, M.D. ${ }^{1,3,4}$, and Kurtis I. Auguste, M.D. ${ }^{1,2,4,5}$ ${ }^{1}$ UCSF Comprehensive Epilepsy Center, University of California, San Francisco

${ }^{2}$ Department of Neurological Surgery, University of California, San Francisco

${ }^{3}$ Department of Neurology, University of California, San Francisco

${ }^{4}$ Department of Pediatrics, University of California, San Francisco

${ }^{5}$ Pediatric Epilepsy Program, Children's Hospital and Research Center Oakland, California

\section{Abstract}

Object-Resection is a safe and effective treatment option for children with pharmacoresistant focal epilepsy, but some patients continue experience seizures after surgery. While most studies of pediatric epilepsy surgery focus on predictors of postoperative seizure outcome, these factors are often not modifiable, and the reasons for surgical failure may remain unclear.

Methods-The authors performed a retrospective cohort study of children and adolescents who received focal resective surgery for pharmacoresistant epilepsy. Both quantitative and qualitative analyses of factors associated with persistent postoperative seizures were conducted.

Results-Records were reviewed from 110 patients, ranging in age from 6 months to 19 years at the time of surgery, who underwent a total of 115 resections. At a mean 3.1-year follow-up, 76\% of patients were free of disabling seizures (Engel Class I outcome). Seizure freedom was predicted by temporal lobe surgery compared with extra-temporal resection, tumor or mesial temporal sclerosis compared with cortical dysplasia or other pathologies, and by a lower preoperative seizure frequency. Factors associated with persistent seizures (Engel Class II-IV outcome) included residual epileptogenic tissue adjacent to the resection cavity (40\%), an additional epileptogenic zone distant from the resection cavity (32\%), and the presence of a hemispheric epilepsy syndrome (28\%).

\section{(C) AANS, 2014}

Address correspondence to: Dario J. Englot, M.D., Ph.D., Department of Neurological Surgery, University of California, San Francisco, 505 Parnassus Ave., Box 0112, San Francisco, CA 94143-0112. englot@ gmail.com.

Disclosure

The authors report no conflict of interest concerning the materials or methods used in this study or the findings specified in this paper. Author contributions to the study and manuscript preparation include the following. Conception and design: Englot. Acquisition of data: Englot, Han, Ivan, Kuperman, Sullivan. Analysis and interpretation of data: Englot, Han, Rolston, Ivan, Chang, Sullivan, Auguste. Drafting the article: Englot. Critically revising the article: Han, Rolston, Chang, Gupta, Sullivan, Auguste. Reviewed submitted version of manuscript: Englot, Rolston, Ivan, Kuperman, Chang, Gupta, Sullivan, Auguste. Approved the final version of the manuscript on behalf of all authors: Englot. Statistical analysis: Englot. Study supervision: Englot, Auguste. 
Conclusions-While seizure outcomes in pediatric epilepsy surgery may be improved by the use of high-resolution neuroimaging and invasive electrographic studies, a more aggressive resection should be considered in certain patients, including hemispherectomy if a hemispheric epilepsy syndrome is suspected. Family counseling regarding treatment expectations is critical, and reoperation may be warranted in select cases.

\section{Keywords}

epilepsy surgery; recurrence; seizure outcome; mesial temporal sclerosis; focal cortical dysplasia

Focal epilepsy is a debilitating yet common neurological disorder in children. Whereas mesial temporal lobe epilepsy (TLE) is the most common epilepsy syndrome in adults, the majority of children with focal epilepsy have a neocortical lesion. Seizures in children with TLE and extratemporal lobe epilepsy are frequently resistant to treatment with antiseizure medications, leading to cognitive and behavioral problems, diminished quality of life, and an increased risk of death. ${ }^{33}$ Patients with pharmacoresistant focal epilepsy may benefit from targeted resection, which results in seizure freedom in approximately three-quarters of children with TLE and one-half of those with extratemporal lobe epilepsy. ${ }^{14,15}$ Because seizure freedom is the most important predictor of quality of life in epilepsy patients, ${ }^{11}$ this is a critical goal in the treatment of epilepsy.

Many studies of epilepsy surgery focus on predictors of seizure outcome. For instance, our group recently performed meta-analyses of reports describing resective surgery for epilepsy in children. We found that seizure freedom is predicted by abnormal MRI in surgery for TLE, and by earlier intervention and localized ictal electroencephalography (EEG) findings in extratemporal lobe epilepsy, whereas epilepsy caused by an identified lesion and lack of generalized seizures predict favorable outcome in both patient populations. ${ }^{14,15}$ Quantitative analysis of seizure outcome predictors is important because it allows improved patient selection and helps guide family discussions, but this approach does have its limitations. Many of these quantitative or categorical predictors cannot be changed (such as age and pathology), while other qualitative factors that may influence outcome are overlooked (such as surgical approach), and postoperative diagnostic studies in patients who continue to have seizures are often not evaluated. Studies that examine outcomes observed after epilepsy surgery often do not identify the reasons that surgery failed, which tends to limit our ability to formulate improved treatment strategies.

In this report, we performed a retrospective cohort study of children and adolescents who received focal resective surgery for pharmacoresistant epilepsy. We completed both quantitative and qualitative analyses of factors associated with persistent seizures after surgery to better understand the reasons for surgical failures in this population, and to help guide treatment strategies going forward. 


\section{Methods}

\section{Patients and Data Collection}

We reviewed the medical records of 130 consecutive focal resections for epilepsy in pediatric patients (ages 0-19 years old). All patients suffered from drug-resistant epilepsy, defined as persistent seizures after at least 2 unsuccessful antiepileptic medication regimens. Surgical cases involving focal lesionectomy, lobar resection, or limited multilobar resection for drug-resistant epilepsy were included, while we excluded functional or anatomical hemispherectomy procedures, disconnection-only procedures (e.g., corpus callosotomy), and device implantations (e.g., vagus nerve stimulator). Surgical procedures were performed between January 1997 and December 2011 at the University of California, San Francisco (UCSF), and between January 2009 and December 2011 at Children's Hospital and Research Center Oakland (CHRCO), California. A minimum of 1 year of postoperative follow-up was required. Fifteen cases that did not have adequate postoperative follow-up were excluded, leaving 115 procedures in 110 patients for subsequent analysis. All study procedures were in compliance with UCSF and CHRCO clinical research policies, and research protocols were approved by the UCSF Committee on Human Research.

Decisions to offer surgery were made by a comprehensive team of pediatric epileptologists, neurosurgeons, neuropsychologists, neuroradiologists, and other practitioners. Standard preoperative evaluation included structural MRI and EEG, and often also included neuropsychological evaluation, PET, and long-term video-EEG monitoring with or without electrocorticography (ECoG) using surgically implanted subdural and/or depth electrodes. Resection was performed by 1 of 5 neurosurgeons, with more than half of the procedures performed by the senior author of the present study (K.I.A.). Resection was customized to incorporate regions of identified epileptogenic zones and/or cerebral lesions, and to preserve eloquent cortex, where applicable. Intraoperative ECoG was used in approximately twothirds of surgeries to further guide resection. Surgical specimens were analyzed by neuropathologists.

All inpatient and outpatient provider notes, laboratory and diagnostic reports, and operative records were reviewed. Demographic and clinical information including patient age, sex, handedness, duration of epilepsy, medication history, surgical history, MRI results, PET results, EEG findings, use of implanted intracranial electrodes for long-term recording, details of the extent of resection, side and lobes of surgery, and the use of intraoperative ECoG were recorded. Pathological diagnoses were obtained from neuropathology reports. The recently modified Palmini classification system was used to classify cases of focal cortical dysplasia (FCD), except in cases in which the pathological description lacked sufficient detail for subclassification. ${ }^{6}$ Details regarding patients' epilepsy history and seizure semiology, including seizure type and frequency, were obtained from preoperative and postoperative assessments by epileptologists. Seizure outcome was determined by evaluating patient status at the latest follow-up evaluation using a modified Engel classification system. ${ }^{12}$ For patients who did not achieve freedom from debilitating seizures postoperatively (Engel Class II-IV outcome), further detailed chart review was performed to qualitatively analyze possible factors contributing to surgical failure. This included a review 
of postoperative diagnostic testing, such as electrophysiology and neuroimaging studies, as well as further surgical procedures undertaken and their respective outcomes.

\section{Statistical Analysis}

To investigate factors associated with seizure outcome (Engel Class I vs II-IV), the chisquare test was used for categorical variables (e.g., sex) and an unpaired Student t-test was used for continuous variables (e.g., age). Prior to utilizing parametric tests, normality of data was verified, and Levene's test for equality of variances was used. For multivariate analysis, variables were entered into a multivariate logistic regression in a backward fashion. Odds ratios were calculated with a 95\% confidence interval. Kaplan-Meier plots were generated for variables found to be significant on multivariate analysis, and were further examined with 2-sided log-rank tests. Statistical significance was assessed at $\mathrm{p}<0.05$ for all tests, and statistical analyses were performed using SPSS (version 20, IBM).

\section{Results}

We reviewed 115 focal resections for epilepsy in 110 patients, ranging in age from 6 months to 19 years (mean 12.3 years) at the time of surgery. Sixty-six patients $(60 \%)$ were male. Sixty patients (54\%) underwent temporal lobe surgery, while the others underwent extratemporal lobe resection. This was the first resective epilepsy procedure for most patients, but 8 patients (7\%) had undergone a previous surgery. Postoperative follow-up duration ranged from 1 to 15 years (mean 3.1 years). Other baseline patient characteristics are summarized in Table 1. As shown in Table 2, a wide spectrum of pathological diagnoses was observed, with malformation of cortical development (MCD), tumor, and mesial temporal sclerosis (MTS) among the most common. Two distinct pathological entities were observed in 11 cases $(9.6 \%)$, while only a single pathology was noted in others.

\section{Seizure Outcomes and Quantitative Analysis of Outcome Predictors}

At the last postoperative follow-up evaluation, 84 patients (76\%) were free of disabling seizures (Engel Class I outcome), including 67 patients (61\%) who achieved complete seizure freedom (Engel Class 1A). Nine individuals (8\%) had an Engel Class II seizure outcome, 8 (7\%) experienced an Engel Class III outcome, and outcome was Engel Class IV in 9 patients (8\%). Accounting for 5 patients who underwent two focal resections included in the present series, 84 (73\%) of 115 surgeries resulted in Engel Class I outcome. While some patients with Engel Class II-IV outcomes experienced a brief period of seizure freedom immediately after surgery, seizures returned in most cases within the first 6 postoperative months.

Seizure outcomes were stratified across various factors of interest to identify potential univariate predictors of favorable seizure outcome (Table 3). While patients who achieved favorable (Engel Class I) seizure outcome had a mean age ( \pm SEM) of $13.3 \pm 0.6$ years at the time of surgery, individuals with persistent postoperative seizures were somewhat younger $(9.4 \pm 1.2$ years, $\mathrm{p}<0.01)$. Also, $87 \%$ of temporal lobe resections, but only $58 \%$ of extratemporal resections, resulted in freedom from disabling seizures $(\mathrm{p}<0.001)$. Although Engel Class I outcome was typical in cases of MTS (100\%) or tumor (91\%), it was less 
often achieved after resection for MCDs such as FCD (51\%), or with other pathologies listed in Table $2(71 \%, p<0.001)$. Finally, preoperative seizure frequency was significantly higher in patients with Engel Class II-IV outcome (39.0 \pm 8.0 seizures per week) compared with those who achieved freedom from disabling seizures $(10.6 \pm 1.8$ seizures per week, $\mathrm{p}<$ 0.001). Other differences examined were not significant (Table 3 ).

As summarized in Table 4, multivariate analysis revealed that Engel Class I outcome was predicted by temporal lobe surgery compared with extratemporal resection, lower frequency of preoperative seizures, and a pathological diagnosis other than MCD. Other variables investigated (Table 3) were not significantly predictive of seizure outcome in multivariate analysis. For illustrative purposes, Kaplan-Meier curves are depicted in Fig. 1 for the variables significantly predicting outcome in multivariate analysis: surgery lobe, pathology, and seizure frequency.

\section{Qualitative Analysis of Seizure Recurrences}

In addition to quantitative analysis of seizure outcome predictors, we performed qualitative analysis of patients with persistent postoperative seizures (Engel Class II-IV outcome) to identify other potential factors contributing to surgical failure. Of the 31 individuals with Engel Class II-IV outcome, further diagnostic workup of persistent seizures was performed in 25 patients by the time of our study. As summarized in Table 5, we identified 1 of 3 primary factors associated with seizure recurrence in these 25 patients, as well as other notable factors in some cases.

In $10(40 \%)$ of 25 cases with seizure recurrence, postoperative electrographic studies suggested residual epileptogenic tissue located adjacent to the resection cavity (Table 5). In 4 of these cases, a risk of residual epileptogenic tissue was expected, as the extent of resection was intentionally limited to avoid eloquent brain regions. In 2 cases, a residual lesion was also observed on postoperative MRI. One illustrative case of a residual epileptogenic tissue involved a 16-year-old right-handed boy with a 14-year history of complex-partial seizures, having undergone 5 unsuccessful antiseizure medications. Seizure semiology and video-EEG monitoring suggested a left occipital epileptogenic zone, and a region of gray-white matter blurring in the mesial left occipital lobe was identified on MRI, suggestive of FCD. The patient underwent left occipital craniotomy with focal cortical resection of the radiological abnormality, which was also the region displaying most frequent epileptiform activity on intraoperative ECoG. Although less frequent epileptiform activity was observed inferior to the surgical target, this cortex was preserved given its normal appearance on MRI, and concern for preserving vision. Postoperatively, the patient experienced no change in seizure frequency (Engel Class IV outcome), and EEG suggested persistent left-occipital seizure origin. Reoperation was performed via left occipital craniotomy 21 months after the initial surgery, and the resection bed was followed inferiorly, to include a gyrus displaying epileptiform activity on intraoperative ECoG. The patient remains seizure free (Engel Class IA outcome) and neurologically intact 2 years after this second surgery, with final pathological analysis demonstrating FCD.

Among 8 (32\%) of 25 cases who experienced persistent seizures, postoperative diagnostic studies suggested the presence of an additional epileptogenic zone, distant from the resection 
cavity (Table 5). Five of these cases were patients with tuberous sclerosis harboring multiple tubers. In 2 cases of lateral temporal epileptogenic zone resection with sparing of the mesial temporal structures, postoperative electrographic and radiological studies suggested the presence of MTS (i.e., dual pathology). One illustrative case of an additional distant epileptogenic zone involved a 15-month-old boy with tuberous sclerosis experiencing numerous daily partial seizures without clear lateralizing seizure semiology despite several antiseizure medication trials. MRI revealed multiple bilateral cortical tubers and subependymal nodules, including a large tuber in the right posterior frontal lobe. VideoEEG monitoring captured 2 seizures originating from the right frontal region, as well as frequent bilateral interictal epileptiform activity. The patient underwent craniotomy and resection of the right frontal tuber with intraoperative ECoG, which demonstrated abnormal electrographic activity most prominent over the target lesion. Postoperatively, the patient experienced a moderate decrease in seizure frequency, but continued to have debilitating epilepsy (Engel Class III outcome). EEG performed after surgery showed bilateral epileptiform activity in the setting of multiple bilateral cortical lesions noted on imaging studies.

Finally, 7 (28\%) of 25 cases with persistent seizures involved patients with a suspected diagnosis of a hemispheric epilepsy syndrome, such as hemimegalencephaly or Rasmussen's encephalitis. In these cases, either targeted resection was planned to avoid potential morbidity associated with hemispherectomy, or a hemispheric epilepsy syndrome diagnosis was uncertain preoperatively. An illustrative case in this category involved a 14-monthold girl with a history of infantile spasms, suffering from 10-12 seizures per day. MRI suggested left hemispheric cortical dysplasia that was most severe in the frontal lobe, with left frontal epileptiform activity noted on EEG and left frontal lobe hypometabolism on PET. She underwent a modified left frontal lobectomy, preserving her primary motor cortex. Intraoperative ECoG showed diffuse epileptiform activity in the frontal lobe before resection, with modest reduction observed on postresection ECoG recordings. Pathology confirmed cortical dysplasia with subcortical white matter hypomyelination. After a few months of seizure freedom, the patient's seizure frequency returned to preoperative baseline levels (Engel Class IV outcome), with epileptiform activity localized to the left hemisphere on EEG. Twenty months after her initial operation, the patient underwent left functional hemispherectomy. At age 5, the patient has been seizure free for 2.5 years (Engel Class IA outcome), with progressive recovery of expected right-sided hemiparesis (able to walk independently and antigravity strength in the right arm), and excellent progress in language function (able to spell her name, count to 40, and speak in 4-word sentences).

\section{Perioperative Adverse Events}

In 115 surgeries, transient postoperative neurological deficits were observed in 9 patients (8\%) who experienced a full recovery. Persistent new hemiparesis was noted after 4 surgeries (3\%), 3 of which were expected given planned resection involving eloquent cortex. Other adverse events occurred in 5 cases (4\%), including 2 wound infections requiring reoperation and intravenous antibiotics, 1 postoperative hematoma requiring evacuation, 1 hospital-acquired pneumonia, and 1 case of postoperative hydrocephalus requiring shunt insertion. There were no cases of perioperative death. 


\section{Discussion}

In the present study, we report a retrospective analysis of factors associated with seizure outcome in focal resective epilepsy surgery in children and adolescents. Favorable Engel Class I seizure outcomes were observed in $76 \%$ of 110 patients, or in $73 \%$ of 115 focal resections (5 individuals underwent two surgical procedures in the study). Consistent with previous reports, there was a higher likelihood of favorable seizure outcome with temporal lobe compared with extratemporal location, tumor, or MTS compared with MCD or other pathologies, and a lower preoperative seizure frequency. Among the 31 patients with persistent seizures after surgery (Engel Class II-IV outcome), sufficient postoperative data were available in 25 cases to identify potential reasons for surgical failure. Factors associated with persistent seizures included residual epileptogenic tissues adjacent to resection cavity (40\%), an additional epileptogenic zone distant from the resection cavity (32\%), and the likely presence of a hemispheric epilepsy syndrome (28\%).

Incomplete resection of an epileptogenic zone occurs by either intentionally limiting the extent of excision to avoid damaging eloquent brain regions or from inadequate delineation of the entire epileptogenic zone. It is of utmost importance to balance the risks of persistent seizures after a conservative resection with that of greater neurological deficits resulting from a more aggressive approach. Fortunately, the majority of neurological deficits present immediately after surgery will improve over time. In one series of tailored rolandic cortex resections reported by de Oliveira and colleagues, while more than $40 \%$ of 48 children experienced postoperative neurological deficits, all recovered fully within 6 months of surgery. ${ }^{10}$ Using functional MRI, Barba and colleagues found that motor function recovery after rolandic epilepsy surgery in two children was associated with reorganization of motorrelated cortical activations, demonstrating the importance of plasticity in postoperative neurological recovery. ${ }^{3}$ Although it is critical to limit the risk of permanent postoperative neurological deficit, it is equally important to consider potentially devastating effects of persistent uncontrolled seizures. Several investigations have shown that children with postoperative seizure freedom enjoy better cognitive function, ${ }^{31,41}$ improved memory, ${ }^{30}$ less need for medication and its effects, ${ }^{26,34}$ fewer behavioral issues, ${ }^{33}$ and enhanced quality of life ${ }^{29,30}$ compared with individuals with continued seizures. At 1 large pediatric epilepsy center, Hemb and co-investigators have suggested that more aggressive resections in their most recent surgical series were associated with improved seizure outcomes without increased morbidity, when compared with a less aggressive approach in older cases. ${ }^{23}$ Thus, in patients with extremely debilitating seizure profiles, or younger individuals with greater potential for neural plasticity and reorganization, an aggressive resection may be warranted.

When incomplete resection of an epileptogenic zone is unintended, what can be done to maximize precise localization of the surgical target? While behavioral seizure semiology, scalp EEG, and PET all help identify the surgical region of interest, accurate delineation of the epileptogenic zone often requires high-resolution diagnostic studies, such as high-field structural MRI and/or invasive electrophysiological recordings. Mounting evidence suggests a greater likelihood of successful epilepsy surgery when a distinct lesion is identified on MRI. ${ }^{9,14,16,47}$ At our institution, we now routinely perform 3-T MRI with epilepsy-specific sequences as part of our preoperative evaluation. In a study performed by Knake and others, 
new lesions were found on 3-T phased-array MR images in 65\% of patients with epilepsy (n $=23$ ) with a reportedly normal 1.5 -T MR image. ${ }^{28}$ The ability to detect subtle epileptogenic lesions will likely be further enhanced as 7-T MRI becomes more widely used in the clinical setting. ${ }^{7}$ Also, in the present study, 2 patients with persistent seizures had a residual lesion identified on postoperative MRI. This finding highlights the importance of achieving grosstotal resection of identified epileptogenic lesions whenever safe and possible.

Except in patients with an unambiguous epileptogenic lesion, we typically use invasive ECoG, either with intraoperative recordings and/or video-EEG monitoring with implanted electrodes, to better delineate the epileptogenic zone. In 1 study by Asano and colleagues describing implanted ECoG in 61 children and young adults, incomplete removal of the area of electrographic abnormality was the only independent predictor of persistent postoperative seizures. ${ }^{2}$ Many other groups have advocated for intraoperative ECoG to guide resection in neocortical epilepsy. ${ }^{20,43,45}$ In the present series, several cases were identified in which intraoperative ECoG helped guide surgical strategy, such as the first illustrative case described in the results, but there was no overall statistical relationship between the use of ECoG and seizure outcomes. Caution is necessary in interpreting this result, however, as cases in which ECoG is used are likely associated a priori with a more difficult epileptogenic zone to localize, a more severe epilepsy, or with a lesion less amenable to complete resection given the involvement of eloquent structures. A prospective, randomized study of the utility of intraoperative ECoG in epilepsy surgery has not yet been performed and is certainly warranted. Continued development of novel strategies for interpreting ECoG signals, including the detection of fast ripple activity ${ }^{1,46}$ and functional connectivity analysis, 5,48 will also be important going forward.

Among the 8 cases in our series in which a separate epileptogenic zone likely contributed to seizure recurrence, 5 patients suffered from tuberous sclerosis. Overall, Engel Class I outcome was achieved in 5 of 10 individuals after resection of an epileptogenic tuber. Bilateral or numerous epileptogenic foci are more common in tuberous sclerosis than other focal epilepsies, especially since there are multiple parenchymal tubers. ${ }^{8,40,44}$ Jansen et al. pooled 177 patients with epilepsy undergoing resective surgery for tuberous sclerosis from 25 articles and found that $57 \%$ of the cohort achieved postoperative seizure freedom. ${ }^{24}$ Similar outcomes were reported in a recent meta-analysis by Fallah and colleagues, who analyzed 181 resections from 20 studies, of which 56\% patients reached an Engel Class I outcome. ${ }^{17}$ The appropriate selection of surgical candidates among patients with tuberous sclerosis requires meticulous preoperative evaluation with high-resolution neuroimaging and electrophysiological recordings, but families should also be counseled that nearly half of these patients continue to have seizures after surgery.

Our study also identified 2 cases of lateral temporal resection in which dual pathology with MTS likely contributed to seizures after surgery. Spencer and colleagues have argued that dual pathology drives ictogenesis in certain cases of TLE, and that cortical dysgenesis, gliosis, and hippocampal sclerosis may allow continued seizures even after gross-total excision of a temporal lobe lesion. ${ }^{18,42}$ Some investigators have argued for more extensive resection in focal epilepsy involving temporal lobe lesions, suggesting that the addition of amygdalohippocampectomy and anterior temporal corticectomy may produce better 
postoperative seizure control than grosstotal lesionectomy alone. ${ }^{13,21,37}$ Thus, in cases of a temporal lobe cortical epileptogenic zone, it is important to rule out dual pathology via careful inspection of seizure semiology and mesial temporal lobe appearance on MRI, and intraoperative ECoG with hippocampal depth electrodes may also be considered. ${ }^{25,27}$ If MTS is present in addition to the cortical lesion, a larger resection including mesial temporal structures may be warranted.

We identified 7 patients with persistent postoperative seizures in the setting of a likely hemispheric epilepsy syndrome. While hemimegalencephaly accounts for $30 \%$ to $50 \%$ of hemispheric epilepsy cases, other causes include Rasmussen's encephalitis, Sturge-Weber syndrome, intracerebral hemorrhage, ischemic stroke, traumatic brain injury, and other forms of MCD. ${ }^{36}$ In 1 case of suspected cortical dysplasia, given both persistent seizures after focal resection and final pathology suggesting Rasmussen's encephalitis, the patient returned for hemispherectomy, ultimately resulting in seizure freedom. In other cases, targeted resection was planned to avoid surgical morbidity associated with hemispherectomy. Compared with focal resection, hemispherectomy is associated with a higher risk of severe hemiparesis, hemianopia, or language dysfunction, and the need for ventriculoperitoneal shunting. ${ }^{19}$ In many cases of suspected hemispheric epilepsy, a challenging decision is whether to pursue focal resection to limit surgical morbidity or proceed directly to hemispherectomy to maximize the chance of seizure freedom (reported as $66 \%$ at 5 years in a recent large singlecenter hemispherectomy series). ${ }^{36}$ Multiple factors must be weighed, including severity of illness, electrographic and radiological findings, patient age and capacity for neural remodeling, baseline neurological deficits, and the product of family discussions.

Finally, in patients with either hemispheric or focal epilepsy syndromes with persistent seizures after surgery, reoperation may be an option. In the present series, Engel Class I outcome was achieved in 7 of 8 patients who underwent repeat focal resection. In a recent series of 23 children undergoing reoperation reported by Ramantani and others, 14 (61\%) were seizure free at last follow-up. ${ }^{39}$ Also, Bauman and colleagues have advocated for a multistage chronic ECoG monitoring approach to failed pediatric epilepsy surgery, which resulted in seizure freedom in $60 \%$ of 15 reoperations in their series. ${ }^{4}$ Considering the debilitating effects of persistent postoperative seizures, further resection should be considered in select patients who fail initial resection.

The present study has several limitations. This is a retrospective cohort investigation, without prospective control, making bias in selection or outcome reporting potential concerns. A significant part of this study involves qualitative assessment of factors contributing to surgical failures, which relies on our subjective interpretation of patient data. Given that only 2 institutions are included in this investigation, results may not fully reflect the population of surgical epilepsy patients. Because we limited the scope of this study to seizure recurrence after targeted resection, neuropsychological and cognitive outcomes are not addressed, yet remain important treatment considerations. Finally, although the longevity of seizure outcomes was also not the focus of this report, we did observe a decrease in seizure freedom rates over time. Others have reported similar seizure recurrences after pediatric epilepsy surgery, ${ }^{30,38}$ although longitudinal studies suggest that early seizure 
freedom is resilient in the majority of cases. ${ }^{35}$ Medication withdrawal and acute postoperative seizures likely only account for a portion of late seizure recurrences. ${ }^{22,32}$ Further study of the reasons for both early and delayed seizure recurrence after pediatric epilepsy surgery remains critically important.

\section{Conclusions}

Focal resection is an effective treatment option for children with pharmacoresistant localizable epilepsy, although some patients continue to experience seizures postoperatively. While seizure outcomes in pediatric epilepsy surgery may be improved by the use of highresolution neuroimaging and invasive electrographic studies, a more aggressive resection should be considered in certain patients, including hemispherectomy if a hemispheric epilepsy syndrome is suspected. The continued pursuit of technological advances in the diagnosis and surgical treatment of pediatric epilepsy surgery is a vital goal, but family counseling regarding the risk of persistent postoperative seizures remains equally important. Reoperation may be warranted in select cases.

\section{Abbreviations used in this paper}

$\begin{array}{ll}\text { CHRCO } & \text { Children's Hospital and Research Center Oakland } \\ \text { ECoG } & \text { electrocorticography } \\ \text { EEG } & \text { electroencephalography } \\ \text { FCD } & \text { focal cortical dysplasia } \\ \text { MCD } & \text { malformation of cortical development } \\ \text { MTS } & \text { mesial temporal sclerosis } \\ \text { TLE } & \text { temporal lobe epilepsy } \\ \text { UCSF } & \text { University of California, San Francisco. }\end{array}$

\section{References}

1. Akiyama T, McCoy B, Go CY, Ochi A, Elliott IM, Akiyama M, et al. Focal resection of fast ripples on extraoperative intracranial EEG improves seizure outcome in pediatric epilepsy. Epilepsia. 2011; 52:1802-1811. [PubMed: 21801168]

2. Asano E, Juhász C, Shah A, Sood S, Chugani HT. Role of subdural electrocorticography in prediction of long-term seizure outcome in epilepsy surgery. Brain. 2009; 132:1038-1047. [PubMed: 19286694]

3. Barba C, Montanaro D, Frijia F, Giordano F, Blümcke I, Genitori L, et al. Focal cortical dysplasia type IIb in the rolandic cortex: functional reorganization after early surgery documented by passive task functional MRI. Epilepsia. 2012; 53:e141-e145. [PubMed: 22686520]

4. Bauman JA, Feoli E, Romanelli P, Doyle WK, Devinsky O, Weiner HL. Multistage epilepsy surgery: safety, efficacy, and utility of a novel approach in pediatric extratemporal epilepsy. Neurosurgery. 2008; 62(Suppl 2):489-505. [PubMed: 18596454]

5. Bettus G, Wendling F, Guye M, Valton L, Régis J, Chauvel P, et al. Enhanced EEG functional connectivity in mesial temporal lobe epilepsy. Epilepsy Res. 2008; 81:58-68. [PubMed: 18547787]

6. Blümcke I, Thom M, Aronica E, Armstrong DD, Vinters HV, Palmini A, et al. The clinicopathologic spectrum of focal cortical dysplasias: a consensus classification proposed by an ad 
hoc Task Force of the ILAE Diagnostic Methods Commission. Epilepsia. 2011; 52:158-174. [PubMed: 21219302]

7. Breyer T, Wanke I, Maderwald S, Woermann FG, Kraff O, Theysohn JM, et al. Imaging of patients with hippocampal sclerosis at 7 Tesla: initial results. Acad Radiol. 2010; 17:421-426. [PubMed: 20018529]

8. Carlson C, Teutonico F, Elliott RE, Moshel YA, LaJoie J, Miles D, et al. Bilateral invasive electroencephalography in patients with tuberous sclerosis complex: a path to surgery? Clinical article. J Neurosurg Pediatr. 2011; 7:421-430. [PubMed: 21456917]

9. Chang EF, Wang DD, Barkovich AJ, Tihan T, Auguste KI, Sullivan JE, et al. Predictors of seizure freedom after surgery for malformations of cortical development. Ann Neurol. 2011; 70:151-162. [PubMed: 21761443]

10. de Oliveira RS, Santos MV, Terra VC, Sakamoto AC, Machado HR. Tailored resections for intractable rolandic cortex epilepsy in children: a single-center experience with 48 consecutive cases. Childs Nerv Syst. 2011; 27:779-785. [PubMed: 21222213]

11. Elliott I, Kadis DS, Lach L, Olds J, McCleary L, Whiting S, et al. Quality of life in young adults who underwent resective surgery for epilepsy in childhood. Epilepsia. 2012; 53:1577-1586. [PubMed: 22812675]

12. Engel, J., Jr; Van Ness, PC.; Rasmussen, TB.; Ojemann, LM. Outcome with respect to epileptic seizures. In: Engel, J., Jr, editor. Surgical Treatment of the Epilepsies, ed 2. New York: Raven Press; 1993. p. 609-621.

13. Englot DJ, Han SJ, Berger MS, Barbaro NM, Chang EF. Extent of surgical resection predicts seizure freedom in low-grade temporal lobe brain tumors. Neurosurgery. 2012; 70:921-928. [PubMed: 21997540]

14. Englot DJ, Lee AT, Tsai C, Halabi C, Barbaro NM, Auguste KI, et al. Seizure types and frequency in patients who "fail" temporal lobectomy for intractable epilepsy. Neurosurgery. 2013; 73:838844. [PubMed: 23892416]

15. Englot DJ, Rolston JD, Wang DD, Sun PP, Chang EF, Auguste KI. Seizure outcomes after temporal lobectomy in pediatric patients. A systematic review. J Neurosurg Pediatr. 2013; 12:134141. [PubMed: 23768202]

16. Englot DJ, Wang DD, Rolston JD, Shih TT, Chang EF. Rates and predictors of long-term seizure freedom after frontal lobe epilepsy surgery: a systematic review and meta-analysis. Clinical article. J Neurosurg. 2012; 116:1042-1048. [PubMed: 22304450]

17. Fallah A, Guyatt GH, Snead OC III, Ebrahim S, Ibrahim GM, Mansouri A, et al. Predictors of seizure outcomes in children with tuberous sclerosis complex and intractable epilepsy undergoing resective epilepsy surgery: an individual participant data meta-analysis. PLoS ONE. 2013; 8:e53565. [PubMed: 23405072]

18. Fish DR, Spencer SS. Clinical correlations: MRI and EEG. Magn Reson Imaging. 1995; 13:11131117. [PubMed: 8750324]

19. Fountas KN, Smith JR, Robinson JS, Tamburrini G, Pietrini D, Di Rocco C. Anatomical hemispherectomy. Childs Nerv Syst. 2006; 22:982-991. [PubMed: 16810492]

20. Gelinas JN, Battison AW, Smith S, Connolly MB, Steinbok P. Electrocorticography and seizure outcomes in children with lesional epilepsy. Childs Nerv Syst. 2011; 27:381-390. [PubMed: 20857122]

21. Giulioni M, Rubboli G, Marucci G, Martinoni M, Volpi L, Michelucci R, et al. Seizure outcome of epilepsy surgery in focal epilepsies associated with temporomesial glioneuronal tumors: lesionectomy compared with tailored resection. Clinical article. J Neurosurg. 2009; 111:12751282. [PubMed: 19408976]

22. Hauptman JS, Pedram K, Sison CA, Sankar R, Salamon N, Vinters HV, et al. Pediatric epilepsy surgery: long-term 5-year seizure remission and medication use. Neurosurgery. 2012; 71:985-993. [PubMed: 22895408]

23. Hemb M, Velasco TR, Parnes MS, Wu JY, Lerner JT, Matsumoto JH, et al. Improved outcomes in pediatric epilepsy surgery: the UCLA experience, 1986-2008. Neurology. 2010; 74:1768-1775. [PubMed: 20427752] 
24. Jansen FE, van Huffelen AC, Algra A, van Nieuwenhuizen O. Epilepsy surgery in tuberous sclerosis: a systematic review. Epilepsia. 2007; 48:1477-1484. [PubMed: 17484753]

25. Kassiri J, Pugh J, Carline S, Jurasek L, Snyder T, Wheatley M, et al. Depth electrodes in pediatric epilepsy surgery. Can J Neurol Sci. 2013; 40:48-55. [PubMed: 23250127]

26. Keene DL, Loy-English I, Ventureyra EC. Long-term socioeconomic outcome following surgical intervention in the treatment of refractory epilepsy in childhood and adolescence. Childs Nerv Syst. 1998; 14:362-365. [PubMed: 9753401]

27. Kim H, Lee C, Knowlton R, Rozzelle C, Blount JP. Safety and utility of supplemental depth electrodes for localizing the ictal onset zone in pediatric neocortical epilepsy. Clinical article. J Neurosurg Pediatr. 2011; 8:49-56. [PubMed: 21721889]

28. Knake S, Triantafyllou C, Wald LL, Wiggins G, Kirk GP, Larsson PG, et al. 3T phased array MRI improves the presurgical evaluation in focal epilepsies: a prospective study. Neurology. 2005; 65:1026-1031. [PubMed: 16217054]

29. Larysz D, Larysz P, Mandera M. Evaluation of quality of life and clinical status of children operated on for intractable epilepsy. Childs Nerv Syst. 2007; 23:91-97. [PubMed: 17053940]

30. Liang S, Wang S, Zhang J, Ding C, Zhang Z, Fu X, et al. Longterm outcomes of epilepsy surgery in school-aged children with partial epilepsy. Pediatr Neurol. 2012; 47:284-290. [PubMed: 22964443]

31. Liu S, An N, Yang H, Yang M, Hou Z, Liu L, et al. Pediatric intractable epilepsy syndromes: reason for early surgical intervention. Brain Dev. 2007; 29:69-78. [PubMed: 16930902]

32. Mani J, Gupta A, Mascha E, Lachhwani D, Prakash K, Bingaman W, et al. Postoperative seizures after extratemporal resections and hemispherectomy in pediatric epilepsy. Neurology. 2006; 66:1038-1043. [PubMed: 16606916]

33. Mikati MA, Ataya N, Ferzli J, Kurdi R, El-Banna D, Rahi A, et al. Quality of life after surgery for intractable partial epilepsy in children: a cohort study with controls. Epilepsy Res. 2010; 90:207213. [PubMed: 20627662]

34. Mikati MA, Rahi AC, Shamseddine A, Mroueh S, Shoeib H, Comair Y. Marked benefits in physical activity and well-being, but not in functioning domains, 2 years after successful epilepsy surgery in children. Epilepsy Behav. 2008; 12:145-149. [PubMed: 17950673]

35. Mittal S, Montes JL, Farmer JP, Rosenblatt B, Dubeau F, Andermann F, et al. Long-term outcome after surgical treatment of temporal lobe epilepsy in children. J Neurosurg. 2005; 103(5 Suppl): 401-412. [PubMed: 16302611]

36. Moosa AN, Gupta A, Jehi L, Marashly A, Cosmo G, Lachhwani D, et al. Longitudinal seizure outcome and prognostic predictors after hemispherectomy in 170 children. Neurology. 2013; 80:253-260. [PubMed: 23223541]

37. Ogiwara H, Nordli DR, DiPatri AJ, Alden TD, Bowman RM, Tomita T. Pediatric epileptogenic gangliogliomas: seizure outcome and surgical results. Clinical article. J Neurosurg Pediatr. 2010; 5:271-276. [PubMed: 20192644]

38. Phi JH, Cho BK, Wang KC, Lee JY, Hwang YS, Kim KJ, et al. Longitudinal analyses of the surgical outcomes of pediatric epilepsy patients with focal cortical dysplasia. Clinical article. J Neurosurg Pediatr. 2010; 6:49-56. [PubMed: 20593988]

39. Ramantani G, Strobl K, Stathi A, Brandt A, Schubert-Bast S, Wiegand G, et al. Reoperation for refractory epilepsy in childhood: a second chance for selected patients. Neurosurgery. 2013; 73:695-704. [PubMed: 23842559]

40. Romanelli P, Weiner HL, Najjar S, Devinsky O. Bilateral resective epilepsy surgery in a child with tuberous sclerosis: case report. Neurosurgery. 2001; 49:732-735. [PubMed: 11523687]

41. Souza-Oliveira C, Escorsi-Rosset S, Terra VC, Muxfeldt-Bianchin M, Machado HR, Sakamoto AC. Impact of pediatric epilepsy surgery on intellectual efficiency. Rev Neurol. 2012; 54:214220. [PubMed: 22314762]

42. Spencer S, Huh L. Outcomes of epilepsy surgery in adults and children. Lancet Neurol. 2008; 7:525-537. [PubMed: 18485316]

43. Tripathi M, Garg A, Gaikwad S, Bal CS, Chitra S, Prasad K, et al. Intra-operative electrocorticography in lesional epilepsy. Epilepsy Res. 2010; 89:133-141. [PubMed: 20079611] 
44. Westmoreland, B. The electroencephalogram in tuberous sclerosis. In: Rodriguez-Gomez, M.; Sampson, JR.; Whittemore, VH., editors. Tubrous Sclerosis Complex: Developmental Perspectives in Psychiatry, ed 3. New York: Oxford University Press; 1999. p. 63-74.

45. Wray CD, McDaniel SS, Saneto RP, Novotny EJ Jr, Ojemann JG. Is postresective intraoperative electrocorticography predictive of seizure outcomes in children? Clinical article. J Neurosurg Pediatr. 2012; 9:546-551. [PubMed: 22546034]

46. Wu JY, Sankar R, Lerner JT, Matsumoto JH, Vinters HV, Mathern GW. Removing interictal fast ripples on electrocorticography linked with seizure freedom in children. Neurology. 2010; 75:1686-1694. [PubMed: 20926787]

47. Yun CH, Lee SK, Lee SY, Kim KK, Jeong SW, Chung CK. Prognostic factors in neocortical epilepsy surgery: multivariate analysis. Epilepsia. 2006; 47:574-579. [PubMed: 16529624]

48. Zaveri HP, Pincus SM, Goncharova II, Duckrow RB, Spencer DD, Spencer SS. Localizationrelated epilepsy exhibits significant connectivity away from the seizure-onset area. Neuroreport. 2009; 20:891-895. [PubMed: 19424095] 
A

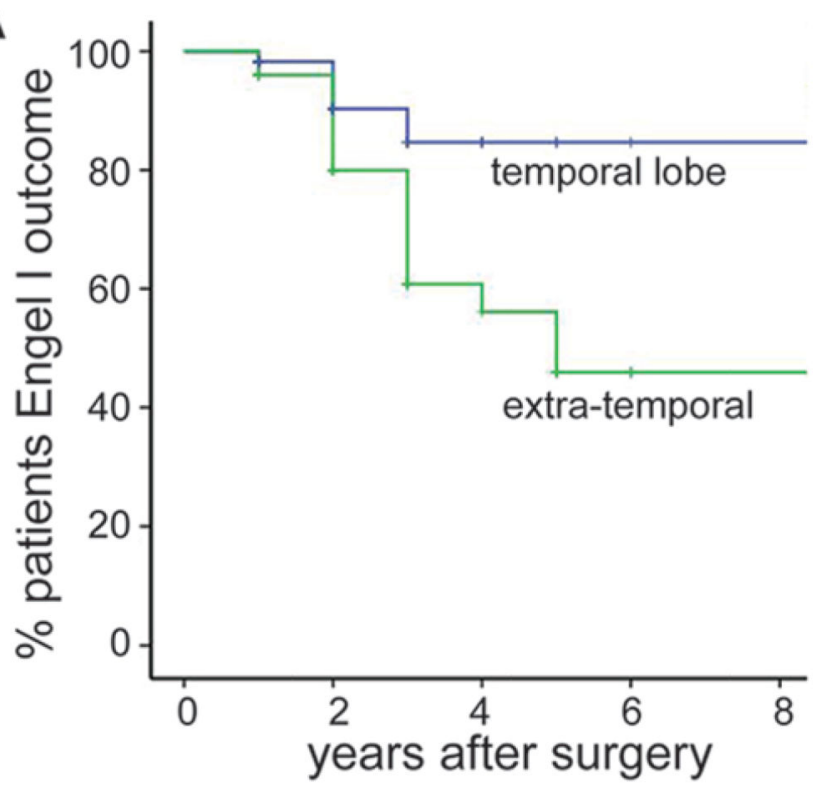

C

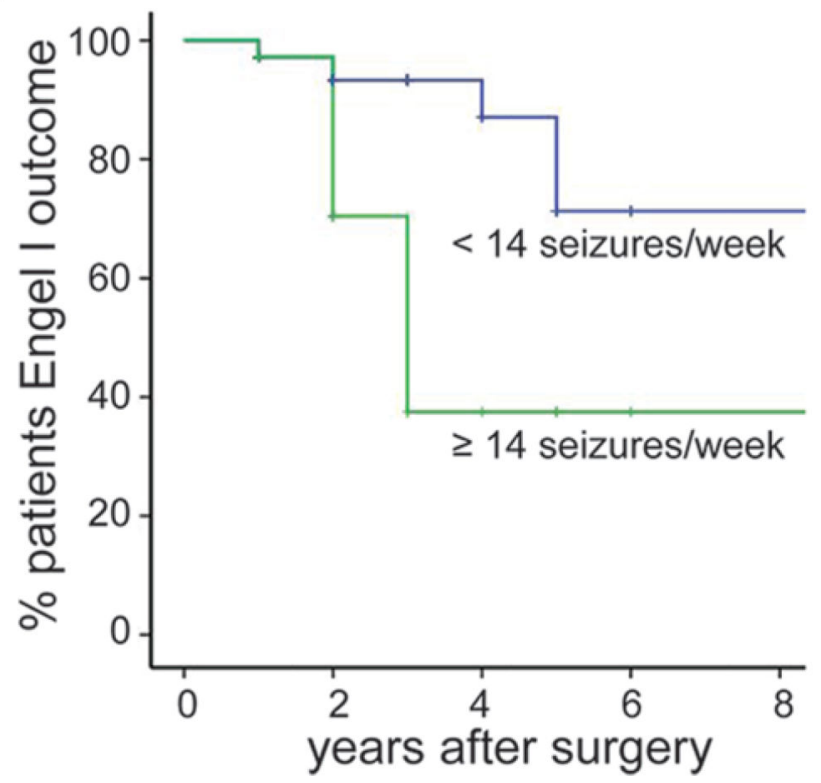

B

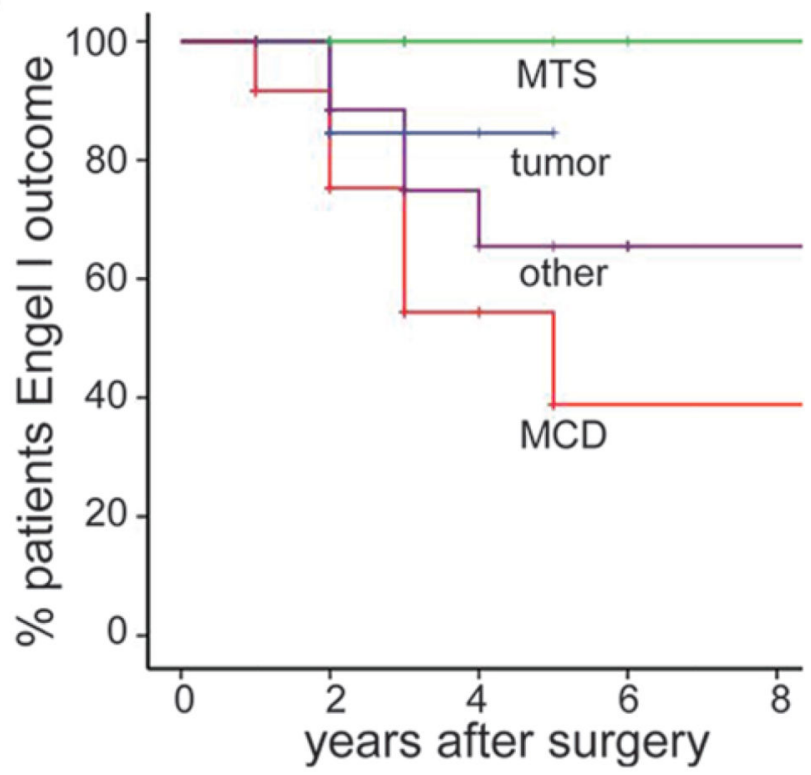

Fig. 1.

Kaplan-Meier plots stratified by significant predictors of seizure outcome. A: Compared with patients who underwent extratemporal lobe epileptogenic zone resection, those who received temporal lobe surgery were more likely to achieve Engel Class I outcome ( $\mathrm{p}<$ 0.05). B: Favorable seizure outcomes were observed in patients with MTS or tumor epilepsy pathology, whereas individuals with MCD were less likely to achieve Engel Class I outcome ( $\mathrm{p}<0.05$ ). C: Patients having fewer than 14 seizures per week preoperatively were more likely to have an Engel Class I outcome than those with higher seizure frequency. Data are 
seizure outcomes (Engel Class I vs II-IV) at last follow-up. Statistical testing was performed with a single 2-sided log-rank test per plot, with significance assessed at $\mathrm{p}<0.05$. 


\section{TABLE 1}

Baseline patient characteristics

\begin{tabular}{|c|c|}
\hline Variable & Value $(\%)$ \\
\hline no. of patients & 110 \\
\hline mean age at surgery \pm SEM (yrs) & $12.3 \pm 0.6$ \\
\hline \multicolumn{2}{|l|}{$\operatorname{sex}$} \\
\hline male & $66(60)$ \\
\hline female & $44(40)$ \\
\hline \multicolumn{2}{|l|}{ handedness } \\
\hline $\mathrm{rt}$ & $59(54)$ \\
\hline lt & $10(9)$ \\
\hline not yet lateralized or unknown & $41(37)$ \\
\hline mean duration of epilepsy \pm SEM (yrs) & $7.0 \pm 0.5$ \\
\hline \multicolumn{2}{|l|}{ lobe involved } \\
\hline temporal & $60(55)$ \\
\hline frontal & $33(30)$ \\
\hline parietal & $5(4)$ \\
\hline occipital & $6(6)$ \\
\hline multiple & $6(6)$ \\
\hline mean no. of seizures/wk \pm SEM & $17.4 \pm 2.8$ \\
\hline \multicolumn{2}{|l|}{ history of generalized seizures } \\
\hline yes & $57(52)$ \\
\hline no & $53(48)$ \\
\hline \multicolumn{2}{|l|}{ side of surgery } \\
\hline $\mathrm{rt}$ & $60(55)$ \\
\hline lt & $50(45)$ \\
\hline \multicolumn{2}{|l|}{ previous resection } \\
\hline no & $102(93)$ \\
\hline yes & $8(7)$ \\
\hline
\end{tabular}

J Neurosurg Pediatr. Author manuscript; available in PMC 2015 October 01. 


\section{TABLE 2}

Pathology in 115 surgeries*

\begin{tabular}{|c|c|}
\hline Pathology & Value \\
\hline \multicolumn{2}{|l|}{ single } \\
\hline \multicolumn{2}{|l|}{ MCD } \\
\hline MCD NOS & 7 \\
\hline FCD Type I & 14 \\
\hline FCD Type IIa & 6 \\
\hline FCD Type IIb & 8 \\
\hline \multicolumn{2}{|l|}{ tumor } \\
\hline ganglioglioma & 7 \\
\hline diffuse astrocytoma & 5 \\
\hline oligodendroglioma & 3 \\
\hline DNET & 1 \\
\hline SEGA & 1 \\
\hline \multicolumn{2}{|l|}{ other } \\
\hline MTS & 17 \\
\hline gliosis only & 17 \\
\hline cortical tuber & 8 \\
\hline ischemia/hypoxia & 4 \\
\hline cavernous malformation & 2 \\
\hline encephalitis & 2 \\
\hline normal brain & 2 \\
\hline \multicolumn{2}{|l|}{ dual } \\
\hline MTS \& encephalitis & 3 \\
\hline FCD Type III \& encephalitis & 2 \\
\hline FCD Type III \& MTS & 1 \\
\hline FCD Type III \& ganglioglioma & 1 \\
\hline FCD Type III \& cortical tuber & 1 \\
\hline FCD Type III \& ischemia/hypoxia & 1 \\
\hline FCD Type III \& cyst & 1 \\
\hline MTS \& ganglioglioma & 1 \\
\hline
\end{tabular}


TABLE 3

Seizure outcomes and associated factors *

\begin{tabular}{|c|c|c|c|}
\hline Variable & Engel Class I (\%) & Engel Class II-IV (\%) & p Value \\
\hline \multicolumn{4}{|l|}{ patient demographics } \\
\hline mean age at surgery & $13.3 \pm 0.6$ & $9.4 \pm 1.2$ & $\operatorname{SEM}(\mathrm{yrs})<0.01^{\dagger}$ \\
\hline \multicolumn{4}{|l|}{ sex } \\
\hline male & $48(69)$ & $22(31)$ & 0.20 \\
\hline female & $36(80)$ & $9(20)$ & \\
\hline \multicolumn{4}{|l|}{ handedness } \\
\hline $\mathrm{rt}$ & $50(81)$ & $12(19)$ & 0.43 \\
\hline lt & $7(70)$ & $3(30)$ & \\
\hline \multicolumn{4}{|l|}{ epilepsy characteristics } \\
\hline \multicolumn{4}{|l|}{ lobe involved } \\
\hline temporal & $52(87)$ & $8(13)$ & $<0.001^{\dagger}$ \\
\hline extratemporal & $32(58)$ & $23(42)$ & \\
\hline \multicolumn{4}{|l|}{ primary pathology } \\
\hline $\mathrm{MCD}$ & $20(51)$ & $19(49)$ & $<0.001^{\dagger}$ \\
\hline tumor & $19(90)$ & $2(10)$ & \\
\hline MTS & $20(100)$ & $0(0)$ & \\
\hline other & $25(71)$ & $10(29)$ & \\
\hline mean duration of epilepsy \pm SEM (yrs) & $7.3 \pm 0.6$ & 6.3. \pm 0.9 & 0.31 \\
\hline mean no. of seizures $/ \mathrm{wk} \pm \mathrm{SEM}$ & $10.6 \pm 1.8$ & $39.0 \pm 8.0$ & $<0.001^{\dagger}$ \\
\hline \multicolumn{4}{|l|}{ history of generalized seizures } \\
\hline yes & $40(67)$ & $20(33)$ & 0.14 \\
\hline no & $44(80)$ & $11(20)$ & \\
\hline \multicolumn{4}{|l|}{ preop diagnostics } \\
\hline \multicolumn{4}{|l|}{ MRI } \\
\hline abnormal & $76(74)$ & $27(26)$ & 0.73 \\
\hline normal & $8(67)$ & $4(33)$ & \\
\hline \multicolumn{4}{|l|}{ scalp ictal EEG } \\
\hline localized & $40(80)$ & $10(20)$ & 0.10 \\
\hline not localized & $44(68)$ & $21(32)$ & \\
\hline \multicolumn{4}{|l|}{ implanted ECoG } \\
\hline performed & $31(72)$ & $12(28)$ & 0.99 \\
\hline not performed & $53(74)$ & $19(26)$ & \\
\hline \multicolumn{4}{|l|}{ PET (when performed) } \\
\hline abnormal & $17(68)$ & $8(32)$ & 0.95 \\
\hline normal & $4(67)$ & $2(33)$ & \\
\hline \multicolumn{4}{|l|}{ operative factors } \\
\hline \multicolumn{4}{|l|}{ side of surgery } \\
\hline $\mathrm{rt}$ & $49(80)$ & $12(20)$ & 0.09 \\
\hline
\end{tabular}




\begin{tabular}{lccc}
\hline Variable & Engel Class I (\%) & Engel Class II-IV (\%) & p Value \\
\hline It & $35(65)$ & $19(35)$ & \\
intraop ECoG & & & \\
performed & $55(71)$ & $22(29)$ & 0.66 \\
not performed & $29(76)$ & $9(24)$ & \\
extent of resection (distinct lesion only) & & & \\
gross-total & $28(78)$ & $8(22)$ & 0.31 \\
subtotal & $3(50)$ & $3(50)$ & \\
previous resection & & & \\
no & $77(72)$ & $30(28)$ & \\
yes & $7(88)$ & $1(12)$ & \\
total & $84(73)$ & $31(27)$ & \\
\hline
\end{tabular}

Note that $\mathrm{n}=115$ surgeries in Table 3 , whereas $\mathrm{n}=110$ patients in Table 1 .

${ }^{\dagger}$ Statistically significant value $(\mathrm{p}<0.05)$ from $\chi^{2}$ test (categorical) or t-test (continuous) comparing patients with Engel Class I versus Class II-IV seizure outcomes. 


\section{TABLE 4}

Predictors of Engel Class I seizure outcome from multivariate logistic regression analysis

\begin{tabular}{lccc}
\hline Predictor & OR $^{*}$ & $\mathbf{9 5 \%}$ CI & p Value \\
\hline temporal lobe surgery & 2.95 & $1.10-7.95$ & $<0.05$ \\
seizure frequency $<14 / \mathrm{wk}$ & 4.46 & $1.66-12.0$ & $<0.01$ \\
MCD & 0.20 & $0.08-0.48$ & $<0.001$ \\
\hline Larger OR indicates higher likelihood of postoperative seizure freedom (Engel Class I outcome).
\end{tabular}


TABLE 5

Qualitative analysis of factors associated with persistent postoperative seizures*

\begin{tabular}{lr}
\hline Primary Associated Factor & No. of Cases \\
\hline residual epileptogenic tissue adjacent to resection cavity & 10 \\
resection intentionally limited to avoid eloquent brain region & 4 \\
residual lesion identified on postop MRI & 2 \\
additional epileptogenic zone distant from resection cavity & 8 \\
multiple tubers in setting of tuberous sclerosis & 5 \\
dual pathology w/ MTS in setting of lateral cortical epileptogenic zone resection & 2 \\
hemispheric epilepsy syndrome & 7 \\
\hline
\end{tabular}

* Of the 31 surgeries associated with Engel Class II-IV seizure outcome, postoperative diagnostic workup was performed in 25 cases. One primary factor was associated with each of these cases, with additional notable factors identified in some cases. 